\title{
INNOVACIÓN, PORTAL HACIA EL FUTURO
}

\author{
LEONIDAS TORRES CITRARO*
}

\section{PARTE I}

\section{INTRODUCCIÓN}

Quiero imaginarme este ensayo como una batería de argumentos para convencer a cualquier oyente sobre la necesidad y las bondades de la innovación, sobre la obligación de integrarla en nuestro ADN, en nuestra cultura, para beneficio de todos los habitantes del país.

La innovación viene siendo estudiada por economistas, educadores y científicos sociales desde hace más de un siglo, sin embargo es en los últimos cincuenta años que notables investigadores han presentado estudios y teorías concretas sobre su naturaleza e importancia.

Dada las limitaciones del ensayo nos restringiremos a presentar una breve reseña de las teorías de tres grandes economistas como son Joseph A. Schumpeter, Robert Solow y Paul Romer: los tres intentan precisar el peso y la influencia de los frutos del conocimiento, es decir la ciencia, tecnología e innovación en el desarrollo de los países y de las empresas.

\footnotetext{
* Ingeniero industrial (1969) de la Universidad de Mackenzie (Sao Paulo, Brasil) e ingeniero mecánico (1988) de la Universidad Central de Venezuela. Asesor empresarial en las áreas de finanzas, inversiones, mercado de capitales y propiedad intelectual. Profesor del posgrado de Propiedad Intelectual de la Universidad de los Andes (Mérida), Venezuela. Contacto: [leonitor@gmail.com]. Fecha de recepción: 14 de marzo de 2016. Fecha de aceptación: 27 de julio de 2016. Para citar el artículo: Torres Citraro, L. Innovación, portal hacia el futuro. Revista La Propiedad Inmaterial n. ${ }^{\circ} 21$, Universidad Externado de Colombia, enero-junio 2016, pp. 111-137. DOI: http://dx.doi.org/10.18601/16571959. n21.05<.
} 


\section{Joseph A. SCHUMPETER}

La destrucción de empresas, fortunas, productos y carreras es el precio del progreso hacia una mejor vida material. Nadie entendía este principio económico mejor que Joseph A. Schumpeter (1883-1950). "Destrucción creativa”, dijo, es la fuerza motriz del capitalismo, del cambio incesante. Su visión era difícil, muchas empresas fracasan, son víctimas de la innovación por parte de sus competidores. Los empresarios ignoran esta lección a su propio riesgo, para sobrevivir tienen que ser emprendedores y pensar estratégicamente. Sin embargo, la opinión de Schumpeter era que la prosperidad general producida por el "motor del capitalismo" es mucho mayor que los restos que deja atrás.

Destrucción creativa es el proceso de innovación que tiene lugar en una economía de mercado en el que los nuevos productos destruyen viejas empresas y modelos de negocio. Para Schumpeter, las innovaciones de los emprendedores son la fuerza que hay detrás de un crecimiento económico sostenido a largo plazo, pese a que puedan destruir en el camino el valor de compañías bien establecidas.

La esencia del capitalismo, señala Schumpeter, es el dinamismo, no el equilibrio estático. Un capitalismo inmóvil sería una contradicción de esos términos. La historia del capitalismo está llena de estallidos violentos y crisis. No es ningún proceso suave de ajuste, sino algo más parecido a una serie de altibajos y acomodos fuertes.

En 1942, Schumpeter bautizó este proceso como "destrucción creativa", la cual es una de las metáforas económicas más exitosas, solamente superada por la "mano invisible" de Adam Smith. El proceso de destrucción creativa, señala Schumpeter, "es el hecho esencial del capitalismo". Su protagonista central es el emprendedor innovador.

Un excelente ejemplo de la validez de los conceptos emitidos por Schumpeter lo encontramos en un interesante informe ${ }^{1}$ de la organización Innosight (2012) donde se analiza la reducción de la vida útil promedio de las empresas que conforman el índice bursátil S\&P500, es decir la longevidad media de las empresas en el S\&P500, que es el índice que incluye a las 500 empresas más valiosas que cotizan en Estados Unidos. Por longevidad media entendemos el número de años que las empresas forman parte del S\&P500, por lo tanto, a más años más estabilidad, y menos años significa que están surgiendo nuevas empresas que las están sustituyendo, que hay una mayor rotación entre los líderes del mercado. Esa longevidad era de 61 años en 1958, se redujo a 25 años en 1980 y para finales del 2012 había bajado a 18 años. En otras palabras en la última década la mitad de las empresas que conformaban dicho índice han sido reemplazadas por nombres y marcas nuevas que copan el paisaje empresarial y nuestra vida cotidiana: Google, Netflix, eBay, Amazon, nvidia, ETrade, Salesforce, Celgene, Facebook, Twitter y muchas otras que no tienen más de veinte años de existencia o que tienen alrededor de diez años, como es el caso de las dos últimas.

1. "Creative Destruction Whips through Corporate America" - Innosight 2012. 
Si quisiéramos tener una perspectiva histórica más amplia, sobre la naturaleza perecedera del liderazgo tecnológico y de la destrucción creativa, basta con recordar los muchos casos de empresas que estuvieron en la vanguardia pero luego se fueron adormeciendo en la engañosa atmósfera del éxito y cuando se percataron habían sido superadas por competidores más inquietos y actualizados. La siguiente lista está conformada por empresas de muy diversos campos, que desaparecieron o fueron absorbidas (muchas formaron parte del S\&P500): Eastman Kodak, Zenith, Polaroid, RCA, Hoover, Compaq, Firestone, PanAm, TwA, American Motors, Blockbuster, Wang Labs, Sun Microsystems, Oldsmobile y Pontiac (ambas de GM), Palm, Merrill Lynch, Lehman Brothers, Bearn Stearns, Arthur Andersen y Enron. En otras palabras, fueron víctimas de la destrucción creativa.

\section{Robert SOLOw}

El investigador Robert Solow fue uno de los primeros en señalar la importancia del conocimiento en el crecimiento económico, expresando que el mismo es una función de capital, trabajo y conocimiento, esto en 1956, cuando publicó $A$ contribution to the economy of economic growth, que fue la base para otorgarle el Premio Nobel de Economía en 1987.

Uno de los aspectos más sorprendentes de su teoría era que la mayor parte del crecimiento de Estados Unidos en la primera mitad del siglo xx no podía ser explicada por el incremento de los factores capital y trabajo. La parte no explicada se denominó "residuo de Solow". Estudios posteriores fueron reduciéndolo, sobre todo al precisar la participación del capital humano; sin embargo, la magnitud del residual siguió siendo considerable.

En general, los resultados de Solow sobre la importancia del residuo no explicado han resistido la prueba del tiempo razonablemente bien. Así, una pieza clave en el crecimiento económico sigue siendo que este no es solo el resultado de los insumos de capital y trabajo, y ni siquiera del capital humano, sino de algo más. No es otra cosa que el "progreso técnico", como lo llamó Solow, o simplemente nuevos conocimientos.

En su estudio, Solow muestra cómo el progreso tecnológico puede ser medido mediante el uso de la función de producción; señala que la mayoría del crecimiento económico de las naciones puede ser atribuible al cambio tecnológico o al crecimiento en la productividad total factorial (PTF), la cual puede ser medida por el residual mediante el acercamiento de la función de producción, y según algunos autores es la medida de nuestra ignorancia.

Uno de los puntos centrales de la economía es cómo los agentes económicos toman los factores de producción como el trabajo, capital e insumos intermedios para convertirlos en productos finales de consumo. A esta relación de factores de producción y producto se le denomina función de producción. Por lo que podemos escribir: 


$$
Y=A f(K, L)
$$

Donde: $Y$ es la producción, $K$ es el stock de capital físico como planta y equipos, $L$ es el trabajo que puede ser medido por el número de horas y el número de personas que trabajan. Mientras que $A$ mide lo que podemos denominar productividad $(f$ significa "en función de"). Un valor mucho mayor de $A$ nos dice que los mismos insumos nos llevan a mayores niveles de producto. Podemos referirnos a $A$ como la productividad total factorial ( $\mathrm{PTF}$ ), que se refiere a mejoras y progreso tecnológico, la invención de nuevas tecnologías o el nivel de destreza de la fuerza laboral en la producción.

La función de producción nos muestra que el producto se incrementa por tres razones fundamentales. Puede ser que las personas trabajen más, lo que significa un mayor nivel de $L$, y ello porque tienen más equipo para trabajar ( $K$ más elevada) o porque el capital y el trabajo se utilizan en la producción de manera más productiva (un nivel de $A$ más elevado).

\section{Paul Romer}

Paul Romer es Senior Fellow en el Centro de Stanford para Desarrollo Internacional (sCid) y en el Instituto Stanford; es el pionero en desarrollar la Teoría del Nuevo Crecimiento, que proporciona una visión nueva sobre cómo los negocios y los gobiernos piensan sobre la creación de riqueza, resaltando la importancia y la fuerza de las nuevas ideas. El término "nuevo" fue utilizado para subrayar que el entendimiento sobre el crecimiento económico pasaba a tener como su motor a los intangibles. Es decir, conceptos como las nuevas ideas, métodos, fórmulas, recetas que permitieron, a lo largo de la historia humana, el crecimiento. Un crecimiento basado en una manera diferente de crear valor.

Romer analiza una de las más viejas preguntas de la economía: ¿qué sostiene el desarrollo económico en un mundo físico caracterizado por la disminución de los recursos y la escasez? La respuesta: la manera en que las sociedades tratan los avances en tecnología.

El desarrollo económico es impulsado por las nuevas ideas y avances en tecnología, y los gobiernos pueden aumentar este índice de crecimiento -de manera que beneficie a todos los ciudadanos-creando incentivos económicos apropiados. En el doctorado que hizo en 1983, Romer argumentó que el descubrimiento científico, el cambio tecnológico, la innovación y el crecimiento del factor productividad total debían ser puestos en el centro del análisis económico y en la médula de la política económica nacional.

Romer estudia cómo las políticas de gobierno afectan a la innovación. Estos nuevos trabajos sugieren que para un país en vías de desarrollo las políticas más importantes del gobierno pueden ser las que determinan el índice de transferencia de tecnología del resto del mundo. Para una economía avanzada, las políticas más 
importantes pueden ser las que influyan en el índice de innovación tecnológica en el sector privado. En 1989 redactó "El cambio tecnológico endógeno"2, trabajo que es lectura de referencia para los estudiosos de la economía. En la introducción el autor señala las siguientes premisas:

El producto por hora trabajada en los Estados Unidos es ahora diez veces más valioso que hace cien años. En los años cincuenta los economistas atribuían casi todo el cambio en el producto por hora trabajada al cambio tecnológico. El análisis subsecuente aumentó nuestras estimaciones de la importancia de los incrementos en la fuerza de trabajo efectiva y en el acervo de capital efectivo para generar crecimiento del producto por trabajador, pero el cambio tecnológico ha sido sin duda de igual importancia. Las materias primas que usamos no han cambiado, pero las instrucciones que seguimos para combinarlas se han tornado mucho más refinadas a resultas de la experiencia, la experimentación, la corrección y la investigación científica. Hace un siglo sólo podíamos obtener un estímulo visual del óxido de hierro usándolo como pigmento. Ahora lo ponemos en una cinta plástica y lo usamos para fabricar grabaciones de videocasetera.

$\mathrm{El}$ argumento presentado en este trabajo se basa en tres premisas. La primera es que el cambio tecnológico - un mejoramiento de las instrucciones para la combinación de las materias primas- se encuentra en la base del crecimiento económico. En consecuencia, el modelo presentado aquí se asemeja al de Solow (1956) con cambio tecnológico. El cambio tecnológico aporta el incentivo necesario para la acumulación continua de capital, y la acumulación de capital y el cambio tecnológico en su conjunto son responsables de gran parte del incremento del producto por hora trabajada.

La segunda premisa dice que el cambio tecnológico surge en gran medida de las acciones intencionales realizadas por personas que responden a los incentivos del mercado. Así pues, el modelo es de cambio tecnológico endógeno más bien que de exógeno. Esto no quiere decir que quien contribuya al cambio tecnológico es motivado por incentivos del mercado. Un científico académico que recibe el apoyo de donativos gubernamentales podría estar totalmente aislado de los incentivos del mercado. La premisa aquí es que los incentivos del mercado desempeñan sin embargo un papel esencial en el proceso por el cual se transforma el conocimiento nuevo en bienes con valor práctico. Nuestro entendimiento inicial del electromagnetismo surgió de la investigación realizada en instituciones académicas, pero la cinta magnética y las grabadoras de videocaseteras hogareñas surgieron de los esfuerzos de empresas privadas para obtener una ganancia.

La tercera de las premisas es que las instrucciones para trabajar con las materias primas son inherentemente distintas de otros bienes económicos. Una vez que se incurre en el costo de crear un nuevo conjunto de instrucciones, éstas pueden usarse una y otra vez sin ningún

2. Romer, Paul. "El cambio tecnológico endógeno”, en El Trimestre Económico, año 1991, vol. LVIII (3), 231, pp. 441-480. 
costo adicional. El desarrollo de instrucciones nuevas y mejores equivale a incurrir en un costo fijo. Se supone que esta propiedad es la característica definitoria de la tecnología.

En un intento de síntesis de los aportes de los tres investigadores reseñados podemos decir que la masa de conocimientos (nuevos y viejos), los cuales deben ser generados por el sistema educativo de cada país, mejorará las habilidades y destrezas de los recursos humanos locales, que conjuntamente con políticas y decisiones acertadas, permitirá iniciar la introducción de innovaciones en productos y procesos, como es el caso de las adaptaciones a las condiciones del mercado local. Sobre esta base se produce un natural aumento de la productividad (proceso que lleva años), condición que luego conduce a un aumento de la competitividad, la cual permite abordar y ganar nuevos mercados, sobre todo si se han alcanzado niveles de calidad para competir en el mercado global. En el diseño de la política de desarrollo, la clase dirigente debe determinar las áreas de fortaleza del país, con el propósito de afirmarlas y robustecerlas con carácter prioritario, dado que es en ellas donde las probabilidades de competir con éxito son mayores. En otras palabras debemos detectar los nichos de mercado donde somos competitivos.

En su publicación ${ }^{3}$ de julio 2015, "El futuro de la productividad", la OECD subraya que "[e]l crecimiento de la productividad es el principal impulsor de los niveles de vida", y más adelante nos recuerda las palabras del economista PAUL Krugman, de acuerdo con el cual la productividad consiste en "trabajar de forma más inteligente, no en trabajar más intensamente", es decir, refleja la capacidad de producir más mejorando la organización de los factores de producción gracias a nuevas ideas, innovaciones tecnológicas y nuevos modelos de negocio.

\section{Parte II}

\section{INTRODUCCIÓN}

En la Parte I esbozamos los principales conceptos y teorías sobre la productividad y el crecimiento, las cuales tratan de medir el peso y la influencia de los frutos del conocimiento, es decir la ciencia, tecnología e innovación en el desarrollo de los países y de las empresas.

Pero si quisiéramos dirigirnos a un público más amplio, para convencerlo de cultivar la innovación, de hacerla parte de su cultura, debemos presentarle situaciones del pasado reciente, tanto para que les asigne su justo valor a las comodidades de hoy, como para que estime lo que cabría esperar en un futuro cercano, con los frutos de las innovaciones que se avizoran, las cuales sin duda mejorarán aún más la calidad de vida de sus usuarios. 
Otro aspecto de suma importancia, como parte del cuerpo de argumentos a presentar a dicho público, es tocar la fibra nacionalista (que sí forma parte de nuestra cultura), mostrando cómo se beneficiarían el país y su población al incorporar la innovación como uno de los pilares de su cultura.

Se ha mencionado ya varias veces el término cultura, por lo que es hora de precisarlo mejor. Decimos que existe una cultura de innovación cuando una comunidad logra elaborar y ofrecer productos, procesos o servicios, que debido a sus conocimientos, experiencias y habilidades presentan un diseño, características o desempeño, además de un precio competitivo y nivel de calidad, que no son superados por otras comunidades.

Un ejemplo es la cultura del vino; se dice que Francia tiene esta cultura, porque en sus provincias sus habitantes han elegido sembrar un tipo de uva (producto de un proceso de ensayo y error) con base en las características de los suelos y el clima de la región. Luego son personas expertas las que deciden cuándo es el momento de la vendimia, para entrar en la etapa de la producción (con muchos detalles y secretos), donde el vino finalizará en unas barricas hechas de maderas seleccionadas e iniciará su proceso de maduración, hasta el momento en que paladares educados señalarán que ya terminó este proceso y se podrá llevar al mercado, donde tendrá aceptación por su calidad y prestigio.

La cultura de innovación es una de mayor complejidad que la del vino, debido a la necesidad de convergencia de muchas variables para que la innovación prospere, siendo los recursos humanos la más importante de ellas. Deben ser personas con conocimientos y experiencias pero con la inquietud y motivación de querer diseñar un nuevo o mejor producto, proceso, servicio o modelo de negocio, con el que se facilite la realización de una tarea o se pueda ejecutar una que antes no era posible, aumentando de esta manera la productividad. Para lograr esa meta, esas personas deberán trabajar con otras en un ambiente que estimule y facilite la innovación, impulsados, no solamente por el eventual beneficio económico que se derive de la innovación, sino por la satisfacción de ver plasmado en ese objeto los conocimientos del equipo en el que trabaja.

Este proceso de transformación, es decir, de establecer una cultura de innovación, solo se puede iniciar una vez que la dirección de la empresa (o los dirigentes del país) ha tomado conciencia de que para sobrevivir se debe innovar. Este convencimiento ha de correr aguas abajo y materializarse mediante decisiones visibles que impacten a sus empleados (o a los ciudadanos), incorporándolos directamente en la construcción del nuevo ambiente innovador. Recordemos que los valores de una empresa (o del país) se transmiten efectivamente más por lo que hacen sus directivos que por lo que dicen.

De aquí en adelante y para una mejor transmisión del mensaje y de los conceptos vamos a dar por entendido que el tema de la innovación se aplica tanto a la empresa como al país; por lo tanto, a la clase dirigente sea de la empresa o del país la designaremos como líderes, facilitando la redacción del mensaje que queremos 
transmitir. Por las mismas razones, cuando se hable de empleados se entenderá que pueden serlo tanto del sector público como del privado.

Para los líderes su conducta incluye mostrar una firme voluntad de sustituir los productos existentes por otros nuevos y mejores, transmitirles energía a los empleados mostrando la disposición de asumir riesgos, experimentar, sembrar la cultura de la innovación y acciones concretas para reducir la burocracia interna, minimizando los trámites para obtener recursos y permisos.

Los empleados que estén dispuestos a asumir el espíritu de innovación deben manifestarlo mediante acciones donde se muestre su tenacidad en resolver los problemas de su proyecto de investigación, el innato deseo de compartir conocimientos así como de buscar apoyo entre los colegas y solicitar recursos por montos factibles, aún más cuando los presupuestos sean estrechos, y escuchar con gran atención a los clientes.

En inglés existe el término "intrapreneurship" para designar la naturaleza de la actividad que desarrolla un empleado dentro de su ámbito de trabajo, donde toma iniciativas y tiene el espíritu del emprendedor, que expone el fruto de su trabajo de innovador a las fuerzas del mercado. La definición tomada de Investopedia. com se traduce como:

Empleado que actúa como un empresario dentro de una organización más grande. El término se deriva de una combinación de "intra" o interno, y "el espíritu empresarial". Los emprendedores (intrapreneurs) suelen ser personas auto-motivadas, proactivas y orientadas a la acción, que se sienten cómodas tomando la iniciativa, incluso dentro de los límites de una organización, en la búsqueda de un producto o servicio innovador.

En todo caso, recordemos el significado de "emprender" según el DRAE: "acometer y comenzar una obra, un negocio, un empeño, especialmente si encierran dificultad o peligro".

Decimos que en la empresa se ha alcanzado un clima de innovación cuando nos sentimos estimulados a experimentar, cuando el ambiente propicia el compromiso con los proyectos en que estamos involucrados, cuando sentimos el desafío de opiniones diferentes a la nuestra, producto de la naturaleza multidisciplinaria de los equipos, cuando se fomenta el aprendizaje permanente y se alienta el pensamiento independiente.

\section{SOBRE EL VALOR DE LA INNOVACIÓN}

\section{A LO LARGO DE LA HISTORIA RECIENTE}

En los primeros años del siglo xIx, Richard Trevithick llevaba a cabo experimentos con motores a vapor con la idea de mejorar el motor de James Watt, que era de baja presión y de muy bajo rendimiento. Como ingeniero de minas en su nativa Cornwall (Reino Unido) había conocido en carne propia la necesidad de bombear 
agua de las minas con rapidez, para lo que se precisaba de un motor más potente y eficiente.

El azar de la vida lo llevó al poblado de Redruth, como vecino de William Murdoch, prestigioso ingeniero e inventor escocés, que había trabajado en la empresa Boulton \& Watt introduciendo notables mejoras en el motor de Watt, como es el caso del engranaje planetario, un mecanismo de conversión de movimiento alternativo a movimiento rotativo. Para el año 1794 Murdoch ya había construido un modelo de un carruaje a vapor y Trevithick lo había visto funcionar.

En la medida que Trevithick acumulaba experiencia, en especial con las calderas, fue desarrollando el motor a vapor de alta presión, que era más pequeño y más potente que los de Watt, siendo que los primeros que construyó eran estacionarios y luego (quizás inspirado por Murdoch) construyó el primer carruaje a vapor en 1801. Con los años, la idea de Trevithick fue perfeccionada por el ingeniero Robert Stephenson quien, junto con su padre George Stephenson, fue vital en el proceso de perfeccionamiento de la locomotora que transitó por la primera línea férrea del mundo entre Liverpool y Manchester, la cual se inauguró el 15 de septiembre 1830. Fue el primer ferrocarril que confió exclusivamente en la energía de vapor, sin la intervención de caballos en trechos empinados, el primero en ser completamente de doble vía en toda su longitud, en tener un sistema de señalización, en utilizar un horario de viajes y en llevar el correo. Los $56 \mathrm{~km}$ de la línea fueron un extraordinario logro de ingeniería para su época, con un túnel de $2.000 \mathrm{~m}$ en el tramo inicial de Liverpool, que fue el primer túnel del mundo excavado debajo de una ciudad y con numerosos viaductos a lo largo de su trazado. La velocidad promedio era de $19 \mathrm{~km} / \mathrm{h}$, debido en buena parte a las limitaciones del propio trazado, alcanzando una máxima de $47 \mathrm{~km} / \mathrm{h}$, desafiando por primera vez al caballo, que era en ese entonces el medio de transporte más rápido y difundido.

La locomotora, como todas las innovaciones, trajo resultados inesperados, y uno de ellos fue la creación de la hora nacional o la estandarización en la medición del tiempo. En los años en que se construyeron las primeras líneas de ferrocarril cada ciudad tenía su hora, no existía una hora nacional; el establecimiento de esta la motivan los horarios de viajes de los trenes, que al fijar una hora de partida y una de llegada entre Liverpool y Manchester, por ejemplo, tenían que referirse a una hora nacional, pues de no ser así la confusión habría sido total. Hacia finales de los años 1840 los británicos lograron la sincronización nacional utilizando para tal fin el telégrafo como medio de comunicación entre las ciudades. Años más tarde se estableció la hora global adoptando el meridiano de Greenwich como meridiano cero, el cual fue aprobado como referencia en una conferencia internacional celebrada en 1884 .

Es oportuno señalar que para el momento en que se inauguró la primera línea férrea del mundo, entre Liverpool y Manchester en 1830, una generación de habilidosos artesanos de Gran Bretaña, como Joseph Bramah (prensa hidráulica), Henry Maudslay, Richard Roberts (taladro múltiple, mejoras en el telar de 
Arkwright), Joseph Whitworth (instrumentos de medición) y James Nasmyth (fresadora, perfiladora y martillo a vapor $)^{4}$, habían concebido y construido las máquinas herramientas que sentaron las bases para el nacimiento de la industria de la ingeniería, que proveyó de maquinarias a toda una gama de industrias aumentando su productividad, lo cual conduciría al dominio del comercio mundial por parte de la Gran Bretaña y su consolidación como primera potencia mundial. Dicha industria de la ingeniería trajo la mecanización general pero en especial la textil, permitió el desarrollo de la red de ferrocarriles, la producción masiva del motor a vapor y la fabricación de los primeros barcos de metal.

Años más tarde, a finales del siglo xIx y principios del siglo xx, la humanidad vivió la gran revolución de la electricidad, que tiene como ícono a Thomas Alva Edison por aquello de la bombilla, pero cuyo mérito en realidad corresponde a una legión de científicos, ingenieros y técnicos que tuvieron que resolver innumerables problemas, además del esfuerzo empresarial y financiero para construir las plantes generadoras y la red eléctrica a lo largo de regiones y países, todo esto para llegar a los hogares donde los primeros y sorprendidos usuarios (alrededor de 1920 en Estados Unidos) conectaban los novedosos dispositivos eléctricos, iniciando la revolución de los electrodomésticos, que estaba encubierta por la revolución de la electricidad. Poco a poco dichos dispositivos fueron invadiendo los hogares, generando cambios sociales radicales al liberar al ama de casa de trabajos esclavizantes. El economista Ha Joon CHANG comenta ${ }^{5}$ datos de la década de 1940 en Estados Unidos que muestran cómo la lavadora de ropa eléctrica redujo el tiempo necesario para limpiar una carga estándar de ropa de cuatro horas a 41 minutos, es decir seis veces menos. La plancha eléctrica también redujo el tiempo necesario para planchar esa carga de ropa de 4,5 horas a 1,75 horas, alrededor de 2,5 veces menos. Estos dispositivos permitieron a las mujeres salir de sus casas e involucrarse en actividades más productivas que lavar la ropa, como es un trabajo remunerado. La llegada de la lavadora y plancha eléctrica como productos de consumo masivo desde mediados del siglo xx contribuyó a un aumento de la participación laboral femenina.

Tener una lavadora de ropa en la casa supone que esta tiene el servicio de agua, pero esto no se empezó a materializar sino a finales del siglo xIx. Tal como lo señalan diversos autores ${ }^{6}$, para 1890 solo el 24\% de los hogares en Estados Unidos disponía de agua corriente, pero esta agua no era potable, dado que no fue sino en septiembre de 1908 que se llevó a cabo la primera desinfección en el suministro de agua corriente al ser tratada con cloro en el acueducto de New Jersey, producto de la investigación realizada por John Laing Leal (1858-1914), médico de Estados Unidos responsable de la concepción de dicha técnica, que al ser aplicada en el resto del mundo salvó a centenares de millones de seres humanos.

4. Mokyr, Joel. "The lever of riches: Technological creativity and economic progress". 5. "23 Things they don't tell you about Capitalism”, Penguin (September 2, 2010), Thing 4 - The washing machine has changed the world more than the internet has.

6. J. Greenwood, A. Seshadri y M. Yorukoglu, "Engines of Liberation", Review of Economic Studies, 2005. 
La facilidad del agua también aplica como un requisito para los retretes o sanitarios, por lo que su presencia en los hogares se inicia a principios del siglo XX, extendiéndose rápidamente por todas las residencias y oficinas de Estados Unidos, como se muestra en la siguiente gráfica.

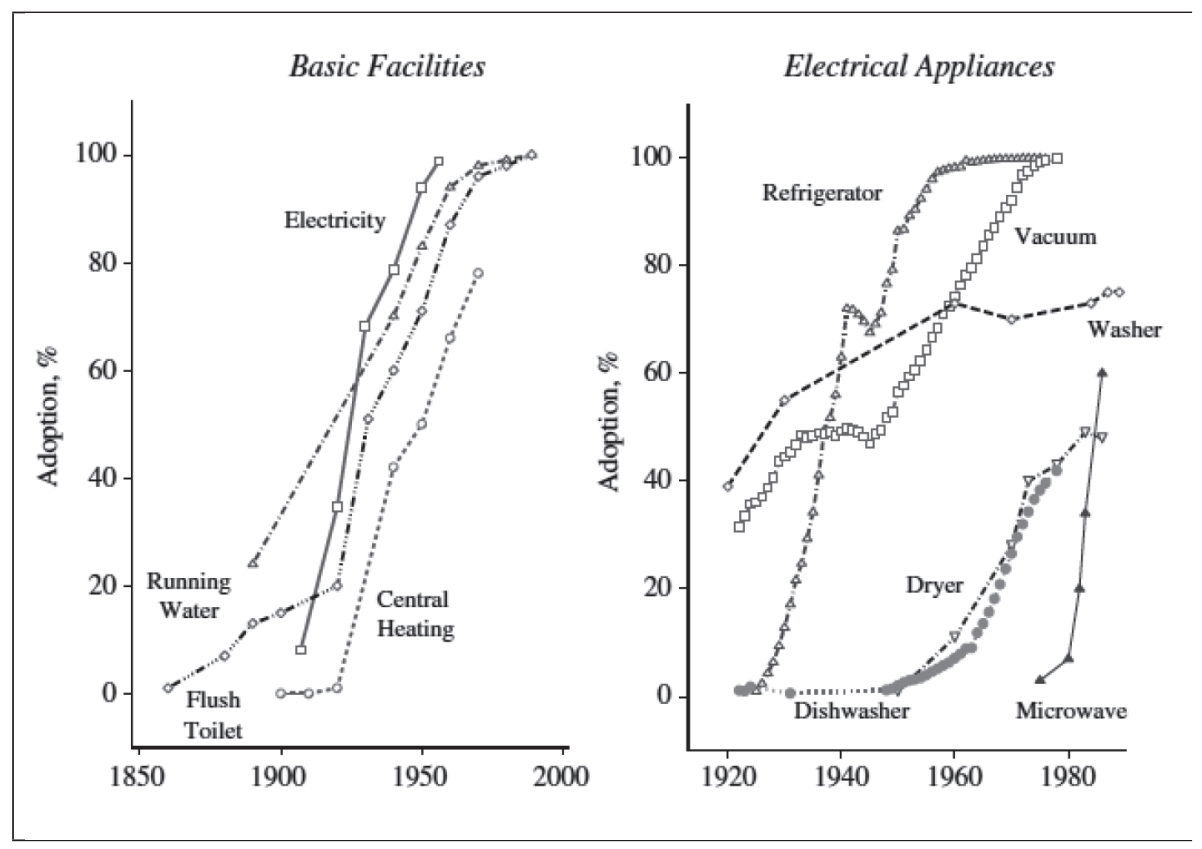

Fuente: J. Greenwood, A. Seshadri y M. Yorukoglu, “Engines of Liberation”, Review of Economic Studies, 2005.

La existencia del conjunto de instalaciones básicas (basic facilities), como son el agua corriente y el agua potable, los retretes, la calefacción central y los sistemas de plomería, es un lugar común en los hogares modernos, pero no era así en los primeros años del siglo xx. Para alcanzar la comodidad de estos servicios se debieron resolver innumerables problemas, introducir mejoras e innovaciones en dichas instalaciones para que operaran satisfactoriamente (confiables, sin fugas) y estandarizar las piezas para facilitar su comercialización y reparación. Todo este esfuerzo nos permite hoy disfrutar de dichas instalaciones, logrando los altos niveles de difusión (adoption) que se observan en el gráfico.

Otro aspecto donde la innovación desempeñó un papel fundamental para la sobrevivencia del género humano como especie son las notables innovaciones que llevaron a mejoras en la eficiencia de procesos industriales y agrícolas a lo largo del siglo xx.

Un primer ejemplo es cómo fueron desmentidas las predicciones apocalípticas anunciadas en las décadas de 1960 y 1970 por prestigiosos investigadores y organizaciones (Paul Ehrlich, The population bomb, 1968; William Paddock, 
Famine 1975, 1968; Club of Rome, The limits to growth, 1972), que advertían de hambrunas debido al crecimiento de la población, al agotamiento de los recursos naturales (petróleo y metales) y a la creciente contaminación. Con lo que no contaban esos profetas del desastre era con iniciativas como la encabezada por Norman Borlaug, conocido como el "Padre de la Revolución Verde", quien ganó el Premio Nobel de la Paz en 1970, al frente de un proyecto que abarcaba el desarrollo de variedades de alto rendimiento de cereales (inicialmente el maíz), el mejoramiento de las técnicas e infraestructura de riego, la modernización de las técnicas de gestión, distribución de semillas, utilización de fertilizantes y pesticidas. Este proyecto contó con el decisivo apoyo de México y la FaO. Otra iniciativa de gran importancia fue el establecimiento del International Rice Research Institute (IRRI) en Filipinas, cuyo objeto central de estudio es el arroz. Se estima que los resultados de la Revolución Verde, al lograr multiplicar tres y cuatro veces el rendimiento de las cosechas, salvaron más de mil millones de personas de la muerte por inanición.

Un segundo ejemplo es la creciente eficacia de los motores de combustión interna, la cual se inició en el año 1973 a raíz del primer gran embargo petrolero, evento económico y político que cuadruplicó los precios del petróleo. Fueron un conjunto de pequeñas innovaciones en los motores con las que se logró un aumento del kilometraje por litro de combustible y una menor contaminación. Pero esto fue parte de una política más amplia que buscaba una mejor utilización de la energía. El próximo gráfico muestra la evolución de la "intensidad energética" en Estados Unidos, concepto que expresa el uso de energía por cada dólar del piB, que nos muestra cómo se va generando más energía con menos dólares:

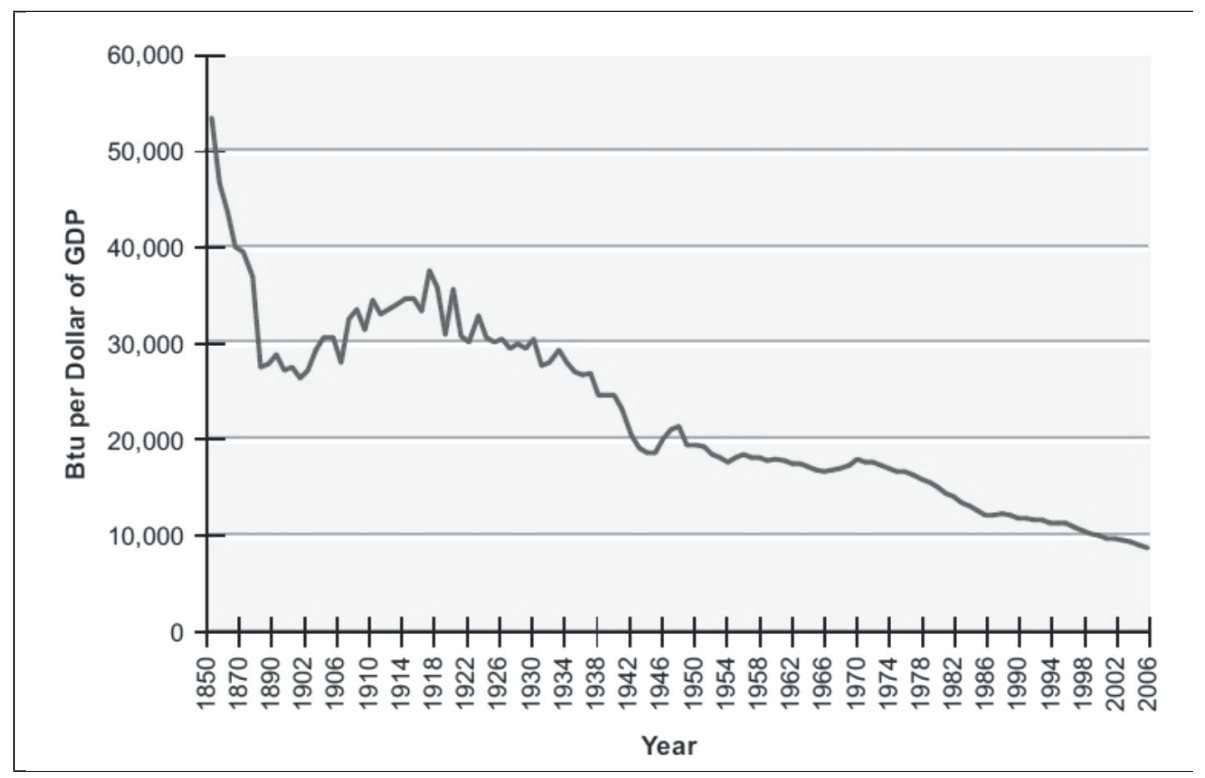

Fuente: Real Prospects for Energy Efficiency in the United States, National Academies Press, 2010. 
La política de ahorro de energía propiciada por el embargo petrolero impulsó a su vez el inicio de innumerables frentes de investigación para perfeccionar innovaciones dirigidas a una mayor eficiencia energética, que en el mediano plazo fue logrando importantes ahorros por medio, entre otros, de los siguientes resultados:

- Con la bombilla incandescente el $10 \%$ de la energía se transforma en luz, mientras que con las lámparas LED lo hace el 50\%. Por otro lado, la iluminación representa casi el $20 \%$ del consumo mundial de electricidad, que es similar a la cantidad de electricidad generada por la energía nuclear. Por lo tanto cualquier innovación en este campo significa un ahorro en energía. Lo anterior de acuerdo con el ya citado Real Prospects for Energy Efficiency in the United States.

- Desde 1960 hemos reducido la cantidad de tierra, agua y energía necesaria para el crecimiento de una fanegada de grano por un factor de 2 a 3, como nos informa el también ya mencionado Ideas on a finite planet de Ramez Naam.

- En 1950 la industria siderúrgica norteamericana utilizaba alrededor de 60 millones de BTU de energía para generar una tonelada de acero, en 1980 era de 30 millones de втU y para 2014 bajó a 12 millones de втU. Este ahorro abarca diferentes industrias, tal como lo muestra el próximo gráfico del Energy Efficiency Index de la industria de la Comunidad Europea, sector responsable del consumo del $26 \%$ de la energía de la región.

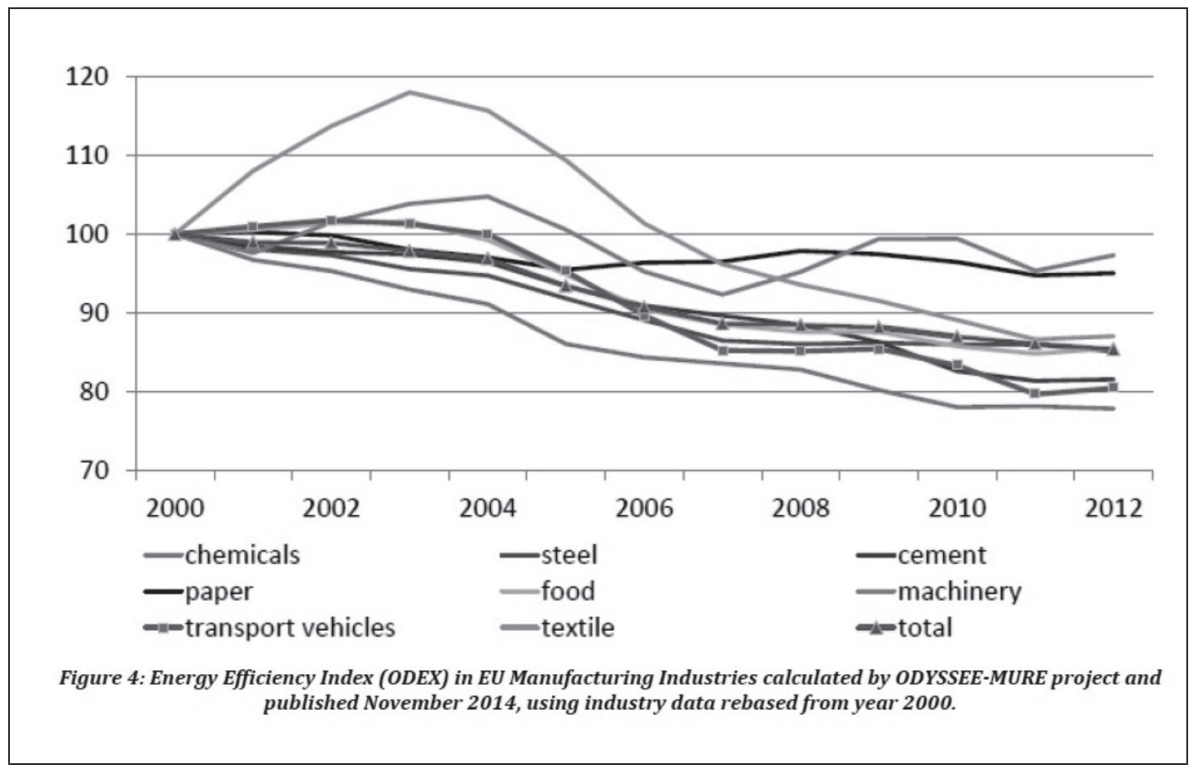

En el gráfico, el índice arranca en año 2000 en 100 puntos, y se puede observar su evolución en los últimos 12 años, con todas las industrias logrando un ahorro que oscila entre el $5 \%$ y el $20 \%$.

- La producción de hierro, acero y aluminio es un proceso muy intensivo en energía, que representan el $10 \%$ del consumo total de energía del sector manu- 
facturero. El reciclaje de dichos metales en el proceso de fabricación ha sido el principal motor de las mejoras en la eficiencia energética en la industria. La producción primaria, en la que el acero está hecho de mineral de hierro y el aluminio a partir del mineral de bauxita, consume mucha energía. Sin embargo, la producción secundaria, que implica el uso de la chatarra de reciclaje para fabricar acero y aluminio, es mucho más eficiente. Se estima que la producción secundaria de acero consume alrededor del $74 \%$ menos de energía que la producción de acero a partir de mineral de hierro, mientras que en la producción de aluminio secundario se requiere un $90 \%$ menos de energía que la producción primaria. La producción secundaria de aluminio representa el 60\% en Estados Unidos, y la producción primaria el restante $40 \%$. Del mismo modo, el reciclado se utiliza en la mayoría de la producción de acero; el 40\% de la producción de acero de Estados Unidos en 2011 procedía de hornos básicos de oxígeno, cuyas entradas son arrabio en casi el $80 \%$, mientras que el $60 \%$ de la producción era de hornos de arco eléctrico, que utilizan más del 90\% de chatarra. Lo anterior de acuerdo con U.S. Energy Information Administration [www.eia.gov/todayinenergy/detail.cfm?id=16211].

Análisis de la Agencia Internacional de la Energía (AIE) muestran que existen oportunidades sustanciales para mejorar la eficiencia energética industrial. El ahorro global potencial de energía en el sector industrial en 2010 asciende a por lo menos 26 EJ (exajoules) por año para el 2030, o el equivalente al consumo de electricidad anual actual de Estados Unidos y China juntos, de conformidad con AIE, "Las 25 recomendaciones de política de eficiencia energética".

Hemos reducido la energía necesaria para mantener fresca la comida en el refrigerador, aun cuando el refrigerador ha crecido en tamaño, si hemos de atender al citado Ideas on a finite planet de Naam.

Desde 1978, la cantidad de energía utilizada para calentar el hogar promedio en Estados Unidos se ha reducido a la mitad (ibíd.).

El transporte aéreo se ha vuelto más eficiente: los aviones a reacción que llegaron al mercado entre 2000 y 2010 son alrededor de 3 veces más eficaces que las aeronaves de la década de 1960. Gracias a mejores motores y una mejor aerodinámica se quema alrededor de un tercio menos de combustible por milla pasajero (ibíd.).

Los edificios tienen un gran potencial de ahorro de energía. La AIE estima que el potencial de ahorro de energía en este sector en 2009 estará en el rango de 20 EJ por año para el 2030, que es equivalente al consumo de electricidad anual actual de Estados Unidos y Japón juntos. Este gran potencial de ahorro de energía se debe en buena parte a las innovaciones de la nanotecnología en el campo de los nuevos materiales, varios de ellos con alta capacidad de aislamiento térmico, que aplicados en edificios significarán menos consumo por calefacción.

En términos de actividad económica, la eficiencia energética puede afectar las tasas de crecimiento del pIB en un rango que va del $0,25 \%$ al $1,1 \%$. En cuanto al empleo, un estudio muestra que cada millón de euros invertido en eficiencia energética crea de 8 a 27 años de trabajo. La situación de la balanza comercial es 
menos clara; la eficiencia energética probablemente creará ganadores y perdedores en el comercio de energía, si se sigue el diagnóstico de AIE en La captura de los múltiples beneficios de la eficiencia energética.

Un ejemplo poco conocido de cómo la innovación actúa en los más insospechados aspectos de la vida es el caso de las ballenas. En el siglo xix el aceite de ballena era la mejor fuente de iluminación en el mundo, producía una llama clara y brillante, con menos humo que el aceite de carbón y menos olor que el aceite de manteca de cerdo. En 1846 la flota ballenera de Estados Unidos tenía 735 barcos y la caza de ballenas era la quinta industria más grande del país. La demanda en Estados Unidos y en el extranjero seguía creciendo. El resultado fue un aumento de los precios: así, en 1820 el aceite de ballena se vendía a US $\$ 200$ por barril, y en su pico de precios, en 1855 , a US $\$ 1.500$ por barril. En 1846 un geólogo canadiense, Abraham Gesner, encontró que si calentaba el carbón seco y luego condensaba el vapor, obtenía un líquido claro que ardía con una llama de calidad limpia y brillante, al que le dio el nombre de querosén. En 1890 la flota ballenera de 735 barcos se había reducido a 65 y el querosén ya disponible en el mercado se vendía a 60 centavos de dólar por galón; fue esta la causa de esa notable reducción de la caza de ballenas y de su salvación como especie. Pero el querosén como principal fuente de iluminación no duraría mucho tiempo: solo entre 1854 y 1890, dado que en 1876 Edison fabricó la primera bombilla incandescente; para finales del siglo XIX el alumbrado eléctrico se iba extendiendo como un reguero de pólvora por toda la geografía de Estados Unidos (cfr. NAAM, Ideas on a finite planet, cit.).

De no haber mediado la Revolución Verde y otras innovaciones en el campo agrícola, el planeta en verdad hubiera vivido grandes hambrunas; de no haber sido por los grandes avances en la salud no tendríamos la serenidad para enfrentar las enfermedades a sabiendas de que las superaremos; de no haber sido por los grandes ahorros logrados en la industria al reciclar componentes, utilizar menos energía e innovar con nuevos materiales, estaríamos generado mayor contaminación, confrontado precios exorbitantes en las materias primas y no disfrutaríamos de las grandes facilidades que nos brindan los artefactos caseros y de oficina; de no haber sido por la lámpara de querosén no existirían esos majestuosos cetáceos en los océanos del planeta.

\section{LA RELACIÓN INDUSTRIALIZACIÓN-CONTAMINACIÓN Y SUS POSIBLES SOLUCIONES}

Por otro lado, no podemos negar el alto grado de contaminación que se ha generado desde el inicio de la Revolución Industrial en el siglo XviII, sin embargo está en manos de la ciencia la posibilidad de detener y disminuir la contaminación. La innovación ha concebido formas de reducir los contaminantes de todo tipo o, en su lugar, reemplazar las sustancias contaminantes con otras que sirven el mismo propósito, pero no dañan el medio ambiente, tal como quedó demostrado en los siguientes casos: 
a) En 1975, el mexicano Mario Molina y el estadounidense Frank Rowland descubrieron que los elementos de los clorofluorocarburos (CFC) no eran tan inertes como se pensaba. Ese mismo año publicaron un artículo en Nature explicando cómo estos compuestos liberados en el aire acababan en las partes altas de la atmósfera, donde la acción de la radiación ultravioleta los descomponía, liberando el cloro. Luego, en una reacción en cadena, el cloro reducía las moléculas de ozono $\left(\mathrm{O}_{3}\right)$ para convertirse en óxido de cloro. Con el agravante de que un solo átomo de cloro puede descomponer 100.000 moléculas de ozono. En mayo de 1985, investigadores británicos anunciaron algo muy preocupante, a saber, que se había abierto un enorme agujero en la capa de ozono sobre la Antártida. Esto produjo una reacción global para buscar soluciones y detener la destrucción de la capa de ozono. De esa preocupación nació el Protocolo de Montreal en 1987, probablemente el mejor ejemplo de cómo la cooperación internacional puede solucionar problemas ambientales globales. Para el momento de la firma de dicho Protocolo no se conocían aún a fondo las causas del debilitamiento de la capa de ozono, pero se actuó por precaución ante lo que sí se sabía. Con los años, el Protocolo acabó siendo firmado por todos los países del planeta. En sucesivas revisiones se ha ido ajustando ante la aparición de nuevos compuestos. Se ha mantenido la prohibición de los CFC y el obligado cumplimiento por parte de los países firmantes. De no haber hecho nada, el agujero sería hoy un $40 \%$ mayor que en 2008, cuando se produjo el pico en su extensión, con unos 25 millones de $\mathrm{Km}^{2}$ de área; también duraría más tiempo y su altura sería mayor. Pero lo más relevante es que no habría un agujero en la capa de ozono, sino dos. Cada año, en el Ártico, también se produce un debilitamiento de la capa de ozono. Según los expertos, en el Ártico el hoyo sería tan grande como hoy lo es en la Antártida. Esta hipotética situación habría incrementado las cifras de cáncer, además de que el exceso de radiación altera procesos básicos para la vida como la fotosíntesis, y en los polos habría acelerado aún más el deshielo.

b) La generación de energía es un aspecto estrechamente vinculado con la contaminación, dado que una buena parte de la energía tiene su origen en los combustibles fósiles (petróleo, carbón, gas). Por eso es importante conocer el esfuerzo que se ha venido realizando en las últimas décadas en el campo de las energías renovables, ya que de su desarrollo depende el futuro de la humanidad. Bajo la Ley de Aire Limpio (Clean Air Act), la agencia epA (Environmental Protection Agency) de Estados Unidos establece los estándares de calidad del aire para proteger la salud pública y el medio ambiente de ese país. La EPA ha establecido normas nacionales de calidad del aire para los seis contaminantes del aire más comunes: monóxido de carbono, ozono, plomo, dióxido de nitrógeno, partículas y dióxido de azufre. Cada año la EPA realiza un seguimiento de los niveles de estos contaminantes en el aire y la cantidad de cada contaminante. La labor de epa se ve en los números que año tras año han mejorado con el tiempo y son publicados en su sitio web. La enmienda de 1970 amplió enormemente el mandato federal, que requiere las regulaciones federales y estatales, tanto para fuentes fijas de contaminación (industrial) como 
para fuentes móviles. Tal como se aprecia en el siguiente gráfico, la lucha contra la contaminación no está disociada del desarrollo; en el gráfico se puede observar el crecimiento de un importante conjunto de variables (PIB, población, consumo de energía) y la reducción en un $69 \%$ de los principales contaminantes.

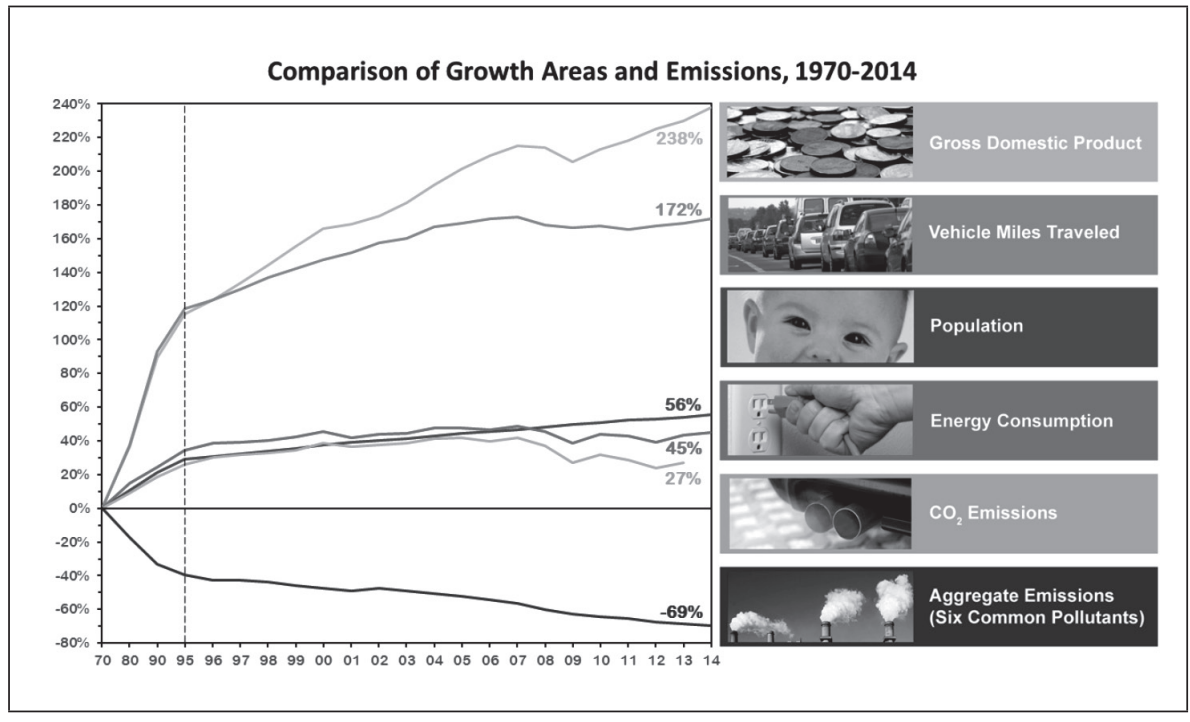

Fuente: [www.epa.gov/clean-air-act-overview].

Un detalle de los principales contaminantes lo podemos apreciar en la siguiente gráfica:

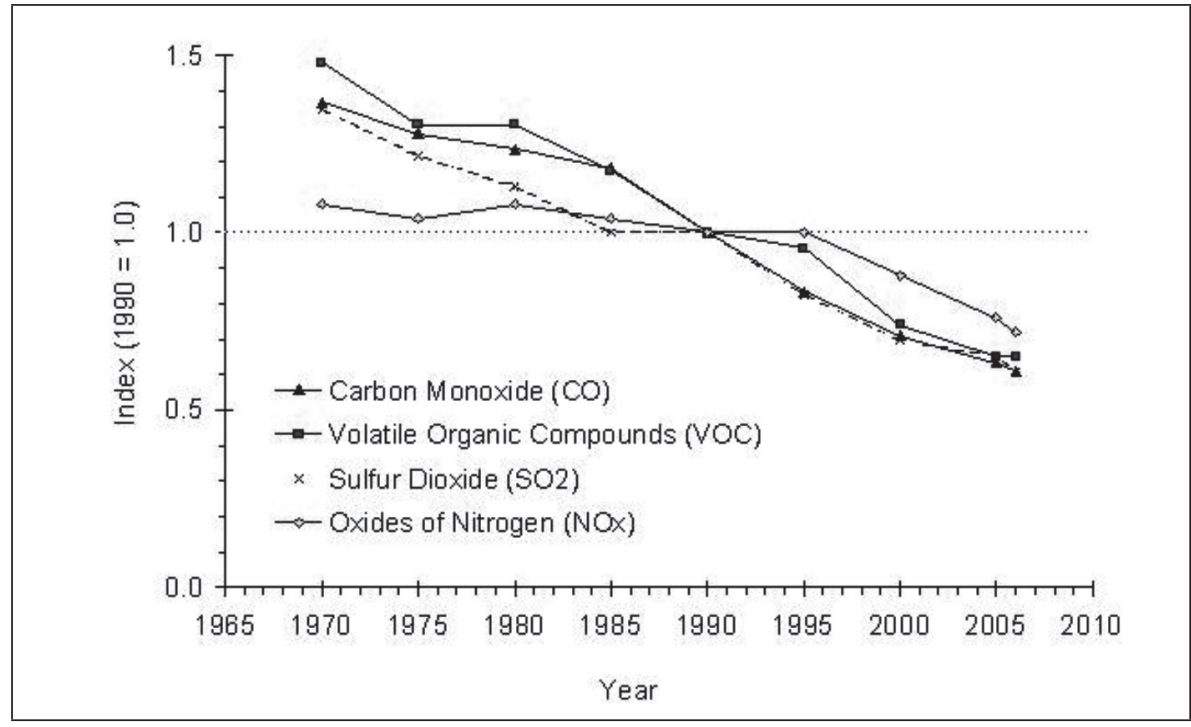

Fuente: Joel Schwartz, World Climate Report, Future air pollutions levels and climate change. 
Podemos observar una fuerte disminución en el período 1965-2010 de contaminantes como el dióxido de sulfuro $\left(\mathrm{SO}_{2}\right)$ causante de la lluvia ácida que estaba afectando los bosques y las aguas de clima templado. Aunque no aparece en el gráfico anterior, también se logró una fuerte disminución, del 98\%, en los niveles de plomo en el período 1980-2014 (cfr. "National trends in lead levels", en [www3. epa.gov/airtrends/lead.html]).

c) En cuanto a las energías renovables, vamos a comenzar con la solar y la eólica, que son las que tienen una mayor cuota de potencia instalada después de la tradicional hidroeléctrica en todo el mundo, así como una mayor tasa de crecimiento, según las cifras ${ }^{7}$ que se presentan a continuación:

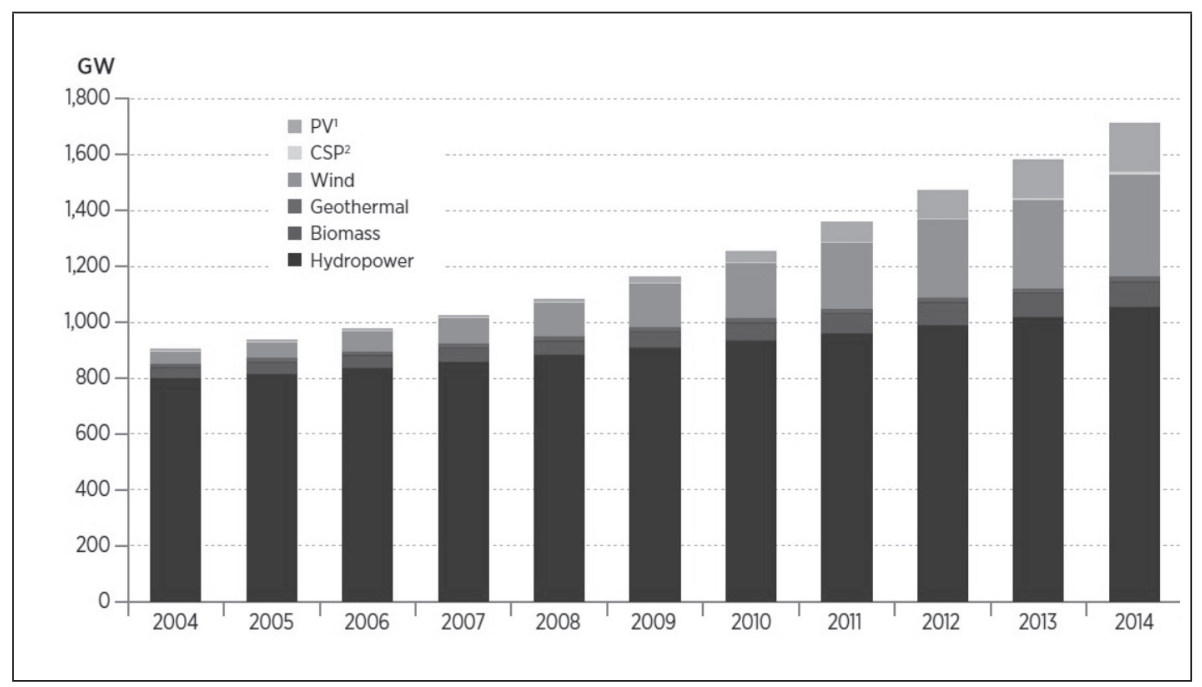

Nota: PV = fotovoltaica (solar) / CSP = concentración por espejos $($ solar $) /$ wind $=$ eólica $/$ geotermal $=$ geotérmica / biomass = biomasa $/$ hydropower $=$ hidroeléctrica.

\begin{tabular}{|l|c|c|c|c|c|}
\hline \multicolumn{7}{|c|}{ Capacidad eléctrica, energías renovables por tecnología } \\
\hline & 2013 & 2014 & 2019 & 2020 & aumento \\
\hline Hidraulica & 1133 & 1168 & 1333 & 1360 & $20 \%$ \\
\hline Bioenergía & 88 & 93 & 125 & 133 & $51 \%$ \\
\hline Eólica & 319 & 362 & 583 & 630 & $97 \%$ \\
\hline Solar & 141 & 181 & 374 & 414 & $194 \%$ \\
\hline Geotérmica & 12 & 12 & 15 & 16 & $33 \%$ \\
\hline Oceánica & 1 & 1 & 1 & 1 & $0 \%$ \\
\hline Total & 1692 & 1817 & 2431 & 2555 & $51 \%$ \\
\hline Cifras en GW & \multicolumn{7}{l}{} \\
\hline Medium-Term Renewable Energy Market Report 2014 - IEA & \\
\hline
\end{tabular}

7. U.S. Department of Energy - National Renewable Energy Laboratory. "2014 Renewable Energy Data Book", November 2015. 
En la proyección que realiza la International Energy Agency (IEA) para el año 2020 se tomó el 2013 como base para calcular el porcentaje de aumento entre ambos años. El aumento en la capacidad de generación eléctrica de la energía eólica es del $97 \%$ y la de energía solar 194\%, que son las modalidades tecnológicas en las que se invierten mayores recursos en I\&D con el propósito de disminuir costos, además de ser respetuosas con el medio ambiente.

Al focalizarnos en primer término en la energía eólica encontramos los siguientes puntos de interés:

- Todos los años se incorporan nuevas instalaciones de generación de energía eléctrica en Estados Unidos, siendo el sector eólico un importante componente desde el año 2007. Como muestra, en el año 2014 la clasificación quedó así: gas (38\%), solar (30\%) y eólica (24\%), cifras que demuestran la creciente importancia de las energías renovables.

- A nivel mundial la capacidad adicional incorporada en el año 2014 fue de $51.000 \mathrm{MW}$, con lo que se superó el récord anterior de $45.000 \mathrm{MW}$, de 2012. Con la cifra de 2014 se alcanzó una capacidad total acumulada de 372.000 MW, distribuidos de acuerdo a la siguiente tabla.

\begin{tabular}{|l|r|l|r|}
\hline \multicolumn{2}{|c|}{ Capacidad anual 2014 en MW } & \multicolumn{2}{c|}{ Capacidad acumulada, final 2014 en MW } \\
\hline China & 23.300 & China & 114.760 \\
\hline Alemania & 5.120 & EE.uU. & 65.880 \\
\hline EE.uU. & 4.850 & Alemania & 39.200 \\
\hline Brasil & 2.780 & India & 22.900 \\
\hline India & 2.300 & España & 22.600 \\
\hline Canadá & 1.870 & Reino Unido & 12.400 \\
\hline Reino Unido & 1.470 & Canadá & 9.690 \\
\hline Suecia & 1.050 & Francia & 9.170 \\
\hline Francia & 1.040 & Italia & 8.550 \\
\hline Turquía & 800 & Brasil & 6.650 \\
\hline Resto del mundo & 6.600 & Resto del mundo & 60.200 \\
\hline Total & 51.200 & Total & 372.000 \\
\hline
\end{tabular}

Fuente: US Department of Energy, 2014 Wind Technologies Market Report.

- Los avances recientes de la tecnología eólica se pueden resumir así: la capacidad media de los aerogeneradores instalados en Estados Unidos en 2014 es de más de 1,9 MW, es decir, un 172\% mayor que los de 1998. La altura media de la caseta de turbinas instaladas en 2014 es de 82,7 metros, un $48 \%$ más que en 1998. Los diámetros medios de rotor de las turbinas eólicas instaladas en 2014 fueron de 99,4 metros, un 108\% mayor que los de 1998, lo que se traduce en un crecimiento del $333 \%$ en el área de barrido del rotor. En cuanto al mercado, las empresas líderes son G\&E, Siemens y Vestas, que capturaron el 98\% del mercado de turbinas de Estados Unidos en 2014, en el siguiente orden: G\&E (60\%), seguido por Siemens 
(26\%) y Vestas (12\%). A nivel mundial, Vestas sigue siendo el principal proveedor, seguido por Siemens, G\&E y Goldwind.

d) La energía solar es una de las modalidades donde más se ha investigado para reducir costos y poder competir con las energías derivadas de combustibles fósiles. En el caso de las células solares fotovoltaicas (CSFv) la investigación ${ }^{8}$ se ha focalizado en la cantidad de silicio utilizada en las CSFV por vatio de potencia eléctrica generada. En el año 2000, por cada vatio de potencia eléctrica que una CSFV producía, su fabricación requería 16 gramos de silicio puro. Hoy en día algunas empresas utilizan menos de 3 gramos de silicio por vatio de potencia eléctrica en sus paneles solares y la tendencia apunta a reducir este insumo por vatio. El uso de menos materias primas y menos energía para la fabricación de las CSFV significa que el retorno de la inversión de la energía solar está mejorando y atrayendo más inversionistas.

A continuación se presenta un gráfico con la tendencia de precios (US\$/KW) en el período 1998-2013, donde se muestra una reducción del 66\% del precio en el período 1998-2013.

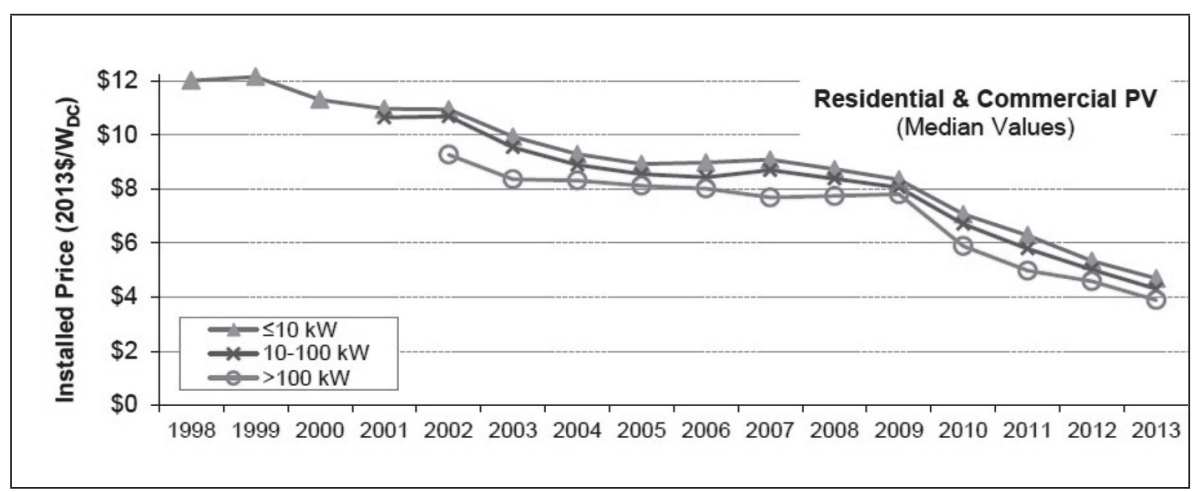

Fuente: Environmental Energy Technologies Division, Lawrence Berkeley National Laboratory, Tracking the Sun VII: An Historical Summary of the Installed Price of Photovoltaics in the United States from 1998 to 2013.

Para medir el impacto ambiental del total de instalaciones de energía solar en el año 2014 se puede decir que estas desplazaron o sustituyeron 20 millones de toneladas métricas de carbón, que equivale a las emisiones de 4 millones de vehículos.

e) Sólo entre el 14\% y el 30\% de la energía del combustible que colocamos en el tanque de un vehículo convencional se utiliza para su desplazamiento. El resto de la energía se pierde en el propio motor y en los varios accesorios de alimentación. Por lo tanto, el potencial de mejora de la eficiencia del combustible con tecnologías avanzadas es enorme. En los vehículos con motor de gasolina, la

8. Environmental Energy Technologies Division, Lawrence Berkeley National Laboratory. "Tracking the Sun vir: An Historical Summary of the Installed Price of Photovoltaics in the United States from 1998 to 2013". 
mayor parte de la energía se pierde en el motor, principalmente en forma de calor (68\%-72\%). Cantidades más pequeñas de energía se pierden en la fricción del motor, la ineficiencia de la combustión, las pérdidas al frenar y la transmisión, la resistencia al viento y el rodamiento de los neumáticos. En el caso de los motores de combustión interna se han logrado importantes mejoras en la eficiencia energética, sin embargo la gran revolución en el transporte está en el vehículo eléctrico y el motor de hidrógeno (fuel cell vehicles), ambos con cero contaminación, de los cuales hay diversos prototipos en fase de prueba (cfr. US Department of Energy, "Fuel economy: Where the energy goes", en [www.fueleconomy.gov/feg/atv.shtml].

f) Finalmente está la bioenergía, sector en el que se han realizado importantes inversiones para la producción de etanol (como sustituto de la gasolina) a partir del maíz, llegando a constituir una significativa parcela del mercado de combustibles en Brasil y Estados Unidos. Sin embargo, la mayor expectativa se ubica en la producción de combustible a partir de las algas, dado que estas ofrecen grandes ventajas: un rendimiento de 3 a 4 veces mayor que el aceite de palma; se requiere poca tierra (no hay que desforestar para su cultivo); demanda poco agua, la cual puede ser no potable y salada; tiene un costo competitivo con otras energías, y los insumos principales son agua, luz solar, $\mathrm{CO}_{2}$ y algo de electricidad. Después de cultivadas, las algas son procesadas y refinadas hasta obtener el aceite biocombustible, quedando un remanente (biomasa) que puede ser utilizado como complemento dietario para animales. Para medir el potencial de las algas se debe considerar que una hectárea de soja produce 450 litros de aceite por hectárea por año, una de girasol 1.000 litros, una de colza 1.200 litros, y la que más da es la destinada a obtener aceite de palma, que provee alrededor de 5 mil litros. El rendimiento de las algas en la actualidad pueden variar de 15 a 30 mil litros de aceite por hectárea por año, dependiendo de la variedad de alga y el sistema de cultivo utilizado, pero de acuerdo a la I\&D en marcha estos números podrían incrementarse. Podríamos estar en presencia de una segunda Revolución Verde con capacidad para enfrentar la creciente demanda de energía de una civilización que albergará cerca de 10.000 millones de habitantes en un futuro cercano (cfr. Instituto de Biotecnología, Universidad Nacional Autónoma de México, Biodiesel a partir de microalgas).

g) Como es bien sabido, la escasez de agua potable es uno de los grandes retos que enfrentamos como especie, al punto que para el año 2025 dos tercios de la humanidad vivirá en zonas que sufrirán la escasez del vital líquido. Por eso, una de las tecnologías más esperanzadoras es la desalinización del agua de mar, cuya gran objeción ha estado referida al alto consumo de energía. Sin embargo innovaciones como el proceso de ósmosis invertida han permitido el establecimiento de grandes plantas desalinizadoras con una fuerte tendencia a un menor consumo de energía, cuya evolución se muestra en el siguiente gráfico. 


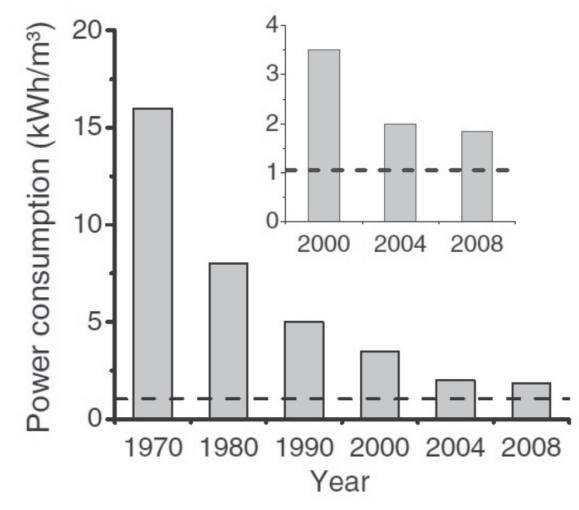

En el período 1970-2008 se obtuvo una reducción, de 16 KWh por m³ , a 1,502,0 KWh por $\mathrm{m}^{3}$, con el resultado de mitigar la crisis de agua potable en muchas regiones del mundo. En el futuro cercano se espera lograr reducciones substanciales con la aplicación de membranas de nanotubos (cfr. Menachem Elimelech y William A. Phillip. "The Future of Seawater Desalination: Energy, Technology, and the Environment", en Science, 2011).

\section{LA DEMANDA ENERGÉTICA FUTURA \\ Y LAS ENERGÍAS RENOVABLES EN COLOMBIA}

La generación de energía es uno de los indicadores que mejor mide el grado de desarrollo de un país, sin ella no podemos proveer los bienes y servicios básicos que en forma creciente demandan las sociedades del siglo xxi. En la medida que un país demanda más energía, significa que sus habitantes son usuarios de artefactos y servicios eléctricos, síntoma de una mejor calidad de vida. También es muy importante el indicador de intensidad energética, que se define como el uso total de energía dividido por el Producto Interno Bruto (PIB), es decir, la cantidad de energía requerida para producir un dólar de PIв. En el caso de los países de la Organización para la Cooperación y el Desarrollo Económicos (OCDE) la intensidad energética es de 130 kilogramos equivalentes de petróleo por dólar, mientras en los países de América Latina y el Caribe es de 230 kilogramos equivalentes de petróleo por dólar; sin lugar a dudas, se tata de un indicador de eficiencia, que demuestra que se produce más con menos energía.

La fuente de las cifras presentadas en los siguientes cuadros es un reciente estudio del Banco Interamericano de Desarrollo, de 2016 y denominado "¿Luces encendidas? - Necesidades de Energía para América Latina y el Caribe al 2040”, donde se reseña el vigoroso crecimiento de la demanda de energía, que se resume con las siguientes cifras: en 1971 la demanda era de 248 millones de toneladas equivalente de petróleo (MTEP), para el año 2013 la demanda subió a 848 MTEP y para el 2040 se estima que llegará a las 1.538 MTEP. 


\begin{tabular}{|l|c|c|c|}
\hline \multicolumn{5}{|c|}{ Uso total de energía al 2013 y 2040 (мтер) } \\
\hline País & 2013 & 2040 & Crecimiento \\
\hline Argentina & 81 & 123 & $52,6 \%$ \\
\hline Brasil & 294 & 577 & $96,6 \%$ \\
\hline Chile & 39 & 99 & $154,7 \%$ \\
\hline Colombia & 32 & 67 & $110,3 \%$ \\
\hline México & 191 & 400 & $109,2 \%$ \\
\hline Venezuela & 69 & 104 & $50,7 \%$ \\
\hline Otros & 144 & 169 & $17,3 \%$ \\
\hline Total ALC & 849 & 1538 & $81,2 \%$ \\
\hline
\end{tabular}

\begin{tabular}{|l|r|r|r|r|r|}
\hline \multicolumn{7}{|c|}{ Matriz energética 1971 y 2013 (MTEP) } \\
\hline & 1971 & & & \multicolumn{1}{c|}{2013} & \\
\hline Carbón & 8,0 & $3,2 \%$ & & 42,8 & $5,0 \%$ \\
\hline Petróleo & 135,9 & $54,7 \%$ & & 389,6 & $45,9 \%$ \\
\hline Gas & 27,2 & $10,9 \%$ & & 199,0 & $23,4 \%$ \\
\hline Nuclear & & & & 8,5 & $1,0 \%$ \\
\hline Hidro & 7,6 & $3,1 \%$ & & 62,8 & $7,4 \%$ \\
\hline Biocombustible & 69,7 & $28,1 \%$ & & 136,7 & $16,1 \%$ \\
\hline Otras renovables & & & & 8,6 & $1,0 \%$ \\
\hline Totales & 248,4 & $100,0 \%$ & & 848,7 & $100,0 \%$ \\
\hline
\end{tabular}

La lectura e interpretación de estas cifras nos debe llamar a la reflexión y a la acción, dado que representan una perspectiva poco halagadora; en primer término, no podemos seguir manteniendo una matriz energética en la que los combustibles fósiles sean los principales componentes, si queremos contribuir a resolver el grave problema del cambio climático. Para el año 1971 el petróleo, el gas y el carbón eran los principales componentes de la matriz energética con el 68,8\%. Para el año 2013 estos tres combustibles fósiles pasan a ser el 74,3\% de dicha matriz.

Por lo tanto, debemos canalizar nuestros esfuerzos de I\&D a las energías renovables, con el propósito de que para el año 2040 estas sean elemento de primera importancia en el suministro de energía de 1.538 MTEP, cifra que representa un $81 \%$ de aumento con relación al año 2013, y alcanzarla representará la inversión de enormes recursos, que cada vez resultan más escasos. Sobre este particular, las energías renovables minimizan la utilización de grandes redes de distribución de electricidad (con grandes inversiones, altos costos de mantenimiento y vulnerabilidad), dado que conceptualmente están diseñadas como pequeños o medianos centros de generación para alimentar comunidades igualmente medianas. Es un concepto diferente de la gran central hidroeléctrica o termoeléctrica, la cual lleva muchos años de construcción y supone enormes recursos, con el propósito de alimentar una región grande, que exige una vasta red de distribución de electricidad; por el contrario, en el caso en estudio 
se trata de pequeñas o medianas granjas eólicas o solares que, además de generar energía para una comunidad específica, deben tener la capacidad de almacenarla. El almacenamiento de energía es uno de los frentes de investigación más activos, debido a su vinculación con la naturaleza intermitente de las energías eólica y solar, las cuales necesitan almacenar en aquellas horas del día en que la generación supera la demanda, para luego poder atender a la comunidad cuando la ecuación se invierte.

En el caso de Colombia se observa un vigoroso crecimiento de la demanda de energía, al pasar de 32 MTEP en el año 2013 a 67 MTEP en el 2040, con un incremento del $110 \%$ entre las dos fechas, por encima del promedio del $81 \%$ de América Latina.

El consumo de energía eléctrica expresado en $\mathrm{kWh} /$ per cápita es uno de los indicadores más reveladores del grado de desarrollo de un país, y consiste en la energía eléctrica consumida por hogares, oficinas e industrias dividido por el número de habitantes. Veamos las estadísticas del siguiente cuadro.

\begin{tabular}{|l|c|}
\hline Consumo de energía eléctrica (kWh per cápita) \\
\hline Chile & 3.865 \\
\hline Venezuela & 3.231 \\
\hline Argentina & 3.175 \\
\hline Brasil & 2.583 \\
\hline México & 2.150 \\
\hline Perú & 1.278 \\
\hline Colombia Key World Energy Statistics 2015 \\
\hline \multicolumn{2}{|c|}{1.153} \\
\hline \multicolumn{2}{|c|}{ International Energy Agency (IEA) } \\
\hline
\end{tabular}

El promedio de consumo de energía eléctrica de este grupo de países de América Latina es de $2.491 \mathrm{kWh} /$ per cápita.

A continuación las estadísticas de un grupo de países desarrollados.

\begin{tabular}{|l|c|}
\hline \multicolumn{2}{|c|}{ Consumo de energía eléctrica (kWh per cápita) } \\
\hline EE.UU & 12.987 \\
\hline Corea del Sur & 10.428 \\
\hline Francia & 7.382 \\
\hline Alemania & 7.022 \\
\hline Holanda & 6.823 \\
\hline Israel & 6.562 \\
\hline España & 5.404 \\
\hline \multicolumn{2}{|c|}{ Key World Energy Statistics 2015 } \\
International Energy Agency (IEA) \\
\hline
\end{tabular}


Al comparar la cifra de Colombia con el promedio de América Latina se obtiene que es el $46 \%$ del mismo, con la de Chile el 30\% y con la de Corea del Sur el $11 \%$, por lo que se concluye que Colombia debe llevar a cabo un ambicioso plan de inversiones en la generación de energía eléctrica, para ir disminuyendo la brecha que la separa de otras naciones vecinas y aún más de los países líderes del mundo.

Creo que el mejor camino que debería tomar América Latina en general, y Colombia en particular, es darle la máxima prioridad y recursos a la I\&D de las energías renovables, tanto para contribuir a enfrentar el delicado problema del cambio climático como para una mejor utilización de sus recursos al escoger la alternativa de un elevado número de centros de generación de energía con redes de distribución locales. Entre los planes debería incluirse el subsidio y financiamiento para la instalación de paneles fotovoltaicos en hogares y edificios, los cuales serían autosuficientes. Lamentablemente, en el "Plan Energético Nacional 'Colombia: Ideario Energético 2050” (Unidad de Planeación Minero Energética, 2015, p. 97) se señala el carácter marginal de las energías renovables, con escasos proyectos en la etapa de factibilidad.

\section{Conclusiones}

A lo largo del artículo vimos que las innovaciones fueron creando el mundo moderno en el que vivimos; cómo el advenimiento de la electricidad y el agua potable alivió a la población de tareas esclavizantes y la libró de terribles epidemias; que las predicciones apocalípticas de prestigiosos investigadores no se materializaron gracias a la labor de otros colegas que elevaron el rendimiento de los cultivos de cereales a niveles nunca vistos, derrotando las hambrunas que amenazaban en el horizonte.

Ante los altos niveles de contaminación y sus indeseables consecuencias, la ciencia, la tecnología y la innovación reaccionaron abordando el tema de la generación de energía -que sin lugar a dudas es la variable fundamental del desarrollo- mediante dos grandes frentes de I\&D: la eficiencia energética y el perfeccionamiento de las energías renovables. Con el primer frente, el objetivo es producir más energía utilizando menos combustible, con un menor grado de contaminación. Respecto de este frente presentamos no menos de diez ejemplos, desde el ahorro logrado con la modesta bombilla hasta la oportuna innovación de la lámpara de querosén, pasando por las notables economías alcanzadas en la producción de hierro, acero y aluminio. Por otro lado, visto el alto grado de contaminación que se ha generado desde el inicio de la Revolución Industrial en el siglo xviıI, señalamos cómo la innovación ha concebido formas de reducir los contaminantes de todo tipo, o en otros casos, de reemplazar dichas sustancias por otras que no dañan el medio ambiente. En otras palabras, tenemos que aceptar que la baja eficiencia energética de los primeros motores y maquinarias generó una alta contaminación, entre otras razones por funcionar con combustibles fósiles. El gran reto de la humanidad para sobrevivir como especie es perfeccionar todas las modalidades de energías 
renovables para, en el menor tiempo posible, llevar a los combustibles fósiles a un uso residual en el museo de la energía.

Para que Colombia construya su portal hacia el futuro y continúe su progreso por la senda del desarrollo debe cubrir las necesidades de energía y electricidad, tarea que implica un reto enorme. Al mismo tiempo, para que Colombia esté en sintonía con su responsabilidad con el equilibrio de los ecosistemas ha de darles prioridad a las energías renovables. Cada fuente de energía plantea importantes impactos medioambientales. Minimizar esos impactos y al mismo tiempo proveer energía asequible y confiable para todos tiene que ser el norte de cualquier plan, el cual necesariamente debe incluir una generosa partida para la $I \& D$ de las energías renovables.

\section{Bibliografia}

Agencia Internacional de la Energía (AIE). "La captura de los múltiples beneficios de la eficiencia energética”, en [www.iea.org/publications/].

Agencia Internacional de la Energía (AIE). "Las 25 recomendaciones de política de eficiencia energética”, en [www.iea.org/publications/].

Banco Interamericano de Desarrollo (2016). "¿Luces encendidas? Necesidades de energía para América Latina y el Caribe al 2040”, en [https://publications. iadb.org/handle/11319/7361?locale-attribute=en].

Chang, H. J. (2010). 23 things they don't tell you about Capitalism, Bloomsbury Press.

Comunidad Europea. Energy Efficiency Index, en [https://ec.europa.eu/energy/en/ topics/energy-efficiency/energy-efficient-products].

Elimelech, M. y W. A. Phillip (August 2011). "The Future of Seawater Desalination: Energy, Technology, and the Environment”, Science Magazine, en [http://science.sciencemag.org/content/333/6043/712].

Environmental Protection Agency. Clean Air Act EPA, en [www.epa.gov/clean-airact-overview].

Greenwood, J.; A. Seshadri y M. Yorukoglu (2005). "Engines of Liberation”. Review of Economic Studies.

Innosight (2012). "Creative Destruction Whips through Corporate America", en [www.innosight.com/innovation-resources/strategy-innovation/creativedestruction-whips-through-corporate-america.cfm].

International Energy Agency (IEA). Key World Energy Statistics 2015, en [www. iea.org/publications/].

Investopedia.com - [www.investopedia.com/].

Lawrence Berkeley National Laboratory. Tracking the Sun vir: An Historical Summary of the Installed Price of Photovoltaics in the United States from 1998 to 2013, Environmental Energy Technologies Division, en [http://eetd.lbl.gov/ sites/all/files/tracking_the_sun_vii_report.pdf]. 
МокуR, J. (1990). The lever of riches: Technological creativity and economic progress, Oxford University Press.

NaAm, R. (2013). The Infinite Resource: The Power of Ideas on a Finite Planet, University Press of New England.

National Academies Press (2010). "Real Prospects for Energy Efficiency in the United States", en [www.nap.edu/].

"National trends in lead levels", en [www3.epa.gov/airtrends/lead.html].

OECD (2015). "El futuro de la productividad", en [www.oecd.org/economy/thefuture-of-productivity.htm].

Romer, P. (1989). "El cambio tecnológico endógeno", El Trimestre Económico, 1991, vol. LVIII (3), n.o 231, pp. 441-480.

Schumpeter, J. (1942). Capitalismo, socialismo y democracia.

SCHWARTZ, J. World Climate Report. Future air pollutions levels and climate change.

Solow, R. (1956). A contribution to the economy of economic growth.

U.S. Department of Energy (November 2015). "2014 Renewable Energy Data Book", National Renewable Energy Laboratory, en [http://energy.gov/eere/ analysis/downloads/2014-renewable-energy-data-book].

U.S. Department of Energy. "Fuel economy: Where the energy goes", en [www. fueleconomy.gov/].

U.S. Department of Energy (2014). Wind Technologies Market Report, en [http:// energy.gov/eere/wind/downloads/2014-wind-technologies-market-report].

U.S. Energy Information Administration, en [www.eia.gov/todayinenergy/].

Universidad Nacional Autónoma de México. "Biodiesel a partir de microalgas", Instituto de Biotecnología, en [www.smbb.com.mx/revista/Revista_2009_3/ Biodiesel.pdf]. 